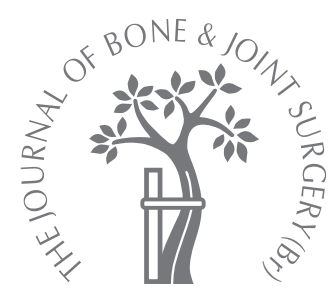

F. O. Abuhassan, A. O. Shannak

From Jordan University Hospital, School of Medicine, Queens Rania Street, Amman 11942, Jordan

\title{
Subperiosteal resection of aneurysmal bone cysts of the distal fibula
}

We describe the treatment by subperiosteal resection of an aneurysmal bone cyst in the distal fibula in eight patients and highlight the role of the periosteum in the regeneration of bone defects. The mean age of the patients was 13.5 years (12 to 17). Seven had an open growth plate. The mean size of the resected specimen was $5.12 \mathrm{~cm}$ (3.5 to 8.0). None of the patients received instillation of bone marrow, autogenous bone graft, allograft or any synthetic bone substitutes.

All had complete regeneration of the bone defect within three to nine months, with no joint instability or recurrence.

The mean length of follow-up was $\mathbf{1 1 . 5}$ years (2 to 18). At the final follow-up there was no difference in the range of movement, alignment or stability of the ankle when compared with the opposite side. The periosteum played a major role in the complete filling of the bone defects and avoided the morbidity of other techniques.

The distal fibula is an uncommon site for an aneurysmal bone cyst. Total resection of the lateral malleolus may produce a deformity and compromise the stability of the ankle. ${ }^{1}$ The main aims of treatment are to eradicate the cyst, avoid local recurrence, prevent damage to the growth plate, avoid subsequent valgus deformity and maintain the stability of the ankle..$^{2-5}$ Curettage and bone grafting have been the usual methods of treatment ${ }^{4}$ for accessible cystic lesions. ${ }^{6,7}$ Various methods have been proposed for the reconstruction of the ankle after total resection of the distal fibula. ${ }^{1,4,5,8}$

The complications of curettage and grafting include a high rate of recurrence, injury to the growth plate, limb-length discrepancy and joint instability. ${ }^{9}$ Resection of the distal fibula for benign bone lesions is an accepted practice, but necessitates reconstruction of the distal tibiofibular joint. It should be reserved for aggressive or recurrent juxtaphyseal lesions, since there is an increased morbidity following multiple procedures, problems with implants and prolonged immobilisation. ${ }^{4}$

We describe our experience of the treatment by subperiosteal resection of an aneurysmal bone cyst of the distal fibula in eight young patients without a bone graft.

\section{Patients and Methods}

There were five girls and three boys with a mean age of 13.5 years (12 to 17 ) who were treated between 1988 and 2006. The lesions were located on the right side in five and on the left in three.

Local pain was the main symptom in all the patients and was associated with unrelated previous injuries in three. Two had a limp and a localised tender mass was palpable in three. The mean duration of symptoms before diagnosis was 4.9 months ( 2 to 7 ). When first seen none of the patients had abnormal alignment or limitation of movement of the ankle. Seven had a visible open growth plate on plain radiographs and in one the growth plate was closed.

Anteroposterior and lateral plain radiographs of the ankle were taken in all the patients. None had MRI or CT. The clinical and radiological details are given in Table $\mathrm{I}$.

Radiological evaluation described the relationship of the cysts to the growth plate and was classified according to the system of Campanacci, Capanna and Picci, ${ }^{10}$ and Capanna et $\mathrm{al}^{11}$ based on the anatomical location. There were five types of lesion with types 1 and 2 corresponding to central forms and types 3 to 5 to peripheral forms. The activity was graded by the system of Capanna et al, ${ }^{11}$ which included three stages, namely aggressive, active and inactive.

The diagnosis was made by the appearance of the fluid drained from the cyst after preoperative aspiration, the typical radiological 
Table I. Clinical and radiological details of the eight patients

\begin{tabular}{|c|c|c|c|c|c|c|}
\hline Case & $\begin{array}{l}\text { Age } \\
\text { (yrs) }\end{array}$ & Gender & $\begin{array}{l}\text { Relation to growth } \\
\text { plate }\end{array}$ & $\begin{array}{l}\text { Size of the resected } \\
\text { specimen }(\mathrm{cm})\end{array}$ & Type of lesion & $\begin{array}{l}\text { Follow-up } \\
\text { (yrs) }\end{array}$ \\
\hline 1 & 12 & $\mathrm{~F}$ & Metaphyseal > $5 \mathrm{~mm}$ & 7.5 & Active & 18 \\
\hline 2 & 12 & $\mathrm{~F}$ & Juxta-epiphyseal & 3.5 & Active & 15 \\
\hline 3 & 17 & $\mathrm{~F}$ & Closed physis & 3.5 & Active & 2 \\
\hline 4 & 14 & $\mathrm{M}$ & Juxta-epiphyseal & 6.0 & Active & 16 \\
\hline 5 & 13 & $\mathrm{M}$ & Juxta-epiphyseal & 8.0 & Active & 18 \\
\hline 6 & 13 & $\mathrm{M}$ & Metaphyseal > $5 \mathrm{~mm}$ & 3.5 & Active & 8 \\
\hline 7 & 14 & $\mathrm{~F}$ & Juxta-epiphyseal & 4.6 & Active & 6 \\
\hline 8 & 13 & $\mathrm{~F}$ & Juxta-epiphyseal & 5.0 & Active & 9 \\
\hline
\end{tabular}

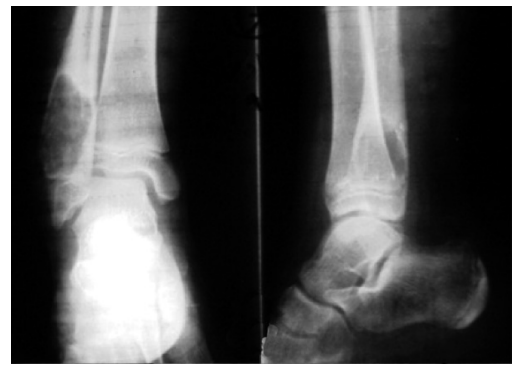

Fig. 1a

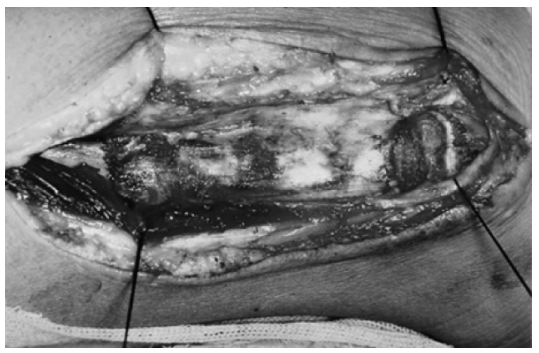

Fig. 1d

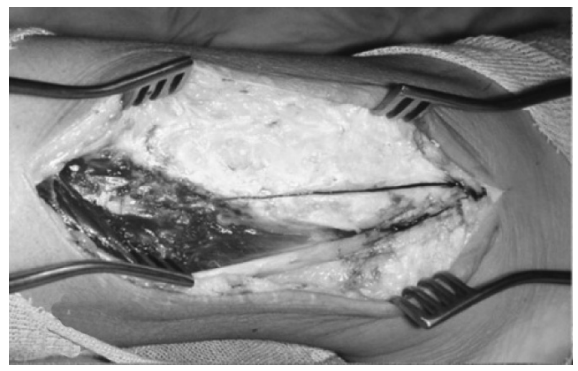

Fig. $1 \mathrm{~b}$

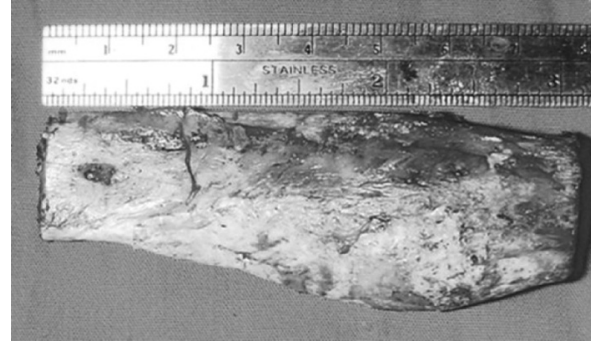

Fig. 1e

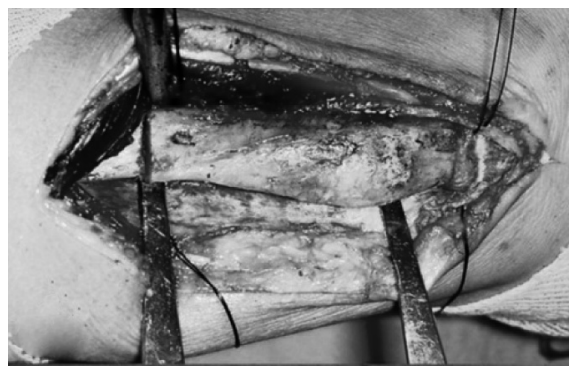

Fig. 1c

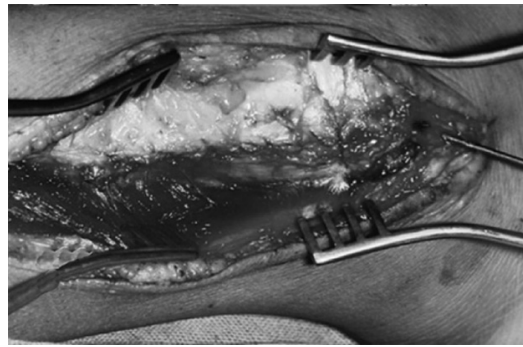

Fig. $1 f$

Case 1. A 12-year-old girl with an aneurysmal bone cyst of the distal fibula. (a) Anteroposterior and lateral plain radiographs showing an expansile lytic lesion of the distal fibula less than $5 \mathrm{~mm}$ from the open growth plate. b) Intra-operative photograph showing the longitudinal incision in the thick periosteum and c) complete peeling of the periosteal sheath with an osteotomy of the lesion proximally and distally and d) the residual defect after complete removal of the lesion. e) Photograph of the resected specimen which measured $7.5 \mathrm{~cm}$ in length. f) Intra-operative photograph showing the Kirschner wire in the distal part of the remaining fibula and the sutures after tight closure of the periosteal sheath.

findings of ballooning of the distal fibula and by histological examination of the resected lesion.

The mean follow-up was 11.5 years ( 2 to 18 ). All the patients had the same surgical procedure of subperiosteal resection without reconstruction. None received instillation of bone marrow, autogenous bone graft, an allograft or any synthetic bone substitutes.

At follow-up clinical and radiological evaluation of the extent of regeneration at the site of resected lesion, physeal growth, alignment, the range of movement of both ankles and recurrence was recorded. Conventional radiography was carried out at four, eight and 12 weeks, at six months and then annually.
Operative technique. Under general anaesthesia with a tourniquet applied to the thigh, the cyst was removed in one piece by subperiosteal resection involving all the cortices without leaving a strut of normal bone.

A lateral longitudinal incision was made over the centre of the lesion down to the periosteum. The periosteal sheath was incised longitudinally and raised gently with a periosteal elevator from the underlying cystic lesion and marked by silk structures (Fig. 1). If the end of the cyst was more than $0.5 \mathrm{~cm}$ from the growth plate, multiple $2.5 \mathrm{~mm}$ diameter drill holes were made away from the growth plate and the bone ends cut by a fine osteotome. The edge of the cyst near the growth plate was removed by a bone nibbler 


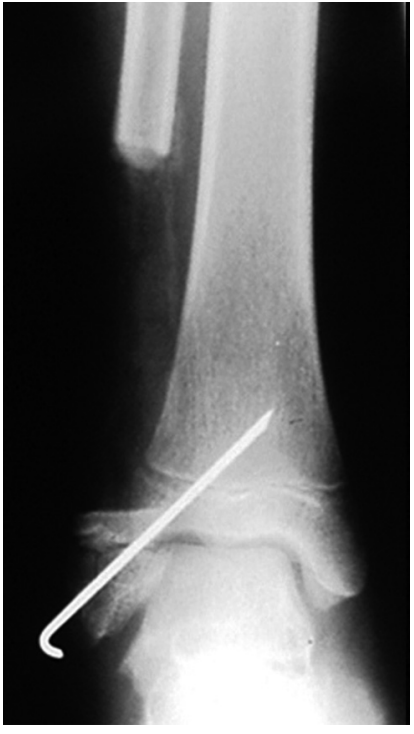

Fig. 2a

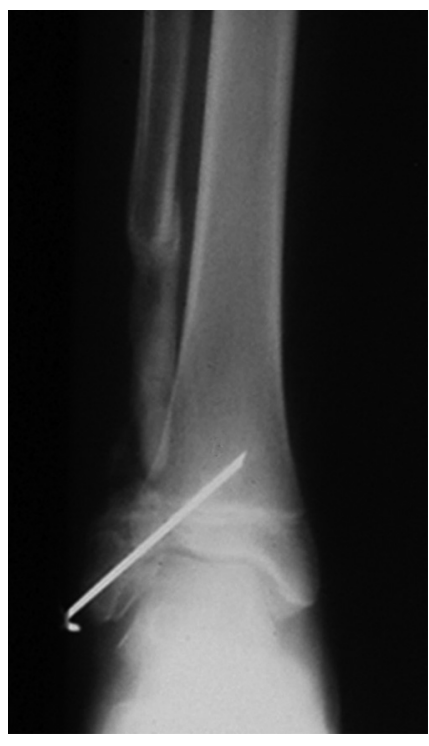

Fig. $2 b$
Case 1. Anteroposterior radiographs showing a) the Kirschner wire holding the lateral malleolus to the tibia with early faint regeneration of new bone in the periosteal tube at three weeks after surgery and b) regeneration two months after surgery before removal of the Kirschner wire.

and a fine curette, with careful preservation of the growth plate. The subperiosteal shell of the bone with the cyst was removed in one piece as far as its attachment to the margins of the remaining normal bone without leaving any bony fragments in the defect. The medullary canal proximal to the remaining bone defect was opened using a $3 \mathrm{~mm}$ Kirschner (K)-wire to allow free communication with the defect. The thick periosteal sheath around the defect was then sutured using 3/0 Vicryl to obtain a tight closure. The remaining distal part of the fibula was fixed to the tibia by a $1.5 \mathrm{~mm} \mathrm{~K}$-wire in all cases in which there was an open growth plate (Fig. 2a).

The skin was closed by subcuticular sutures, with no wound drainage. The limb was immobilised in a plaster cast with partial weight-bearing allowed. After two months the cast and $\mathrm{K}$-wires were removed and the patients were allowed full movement and weight-bearing as tolerated. They were able to participate in sports after six months.

\section{Results}

The mean size of the resected cysts was $5.2 \mathrm{~cm}$ (3.5 to 8.0). Using the classification of Campanacci et $\mathrm{al}^{10}$ all the lesions were type 2 and were active. At the final follow-up there was no difference in the mean range of movement in the ankle compared with that of the opposite side. No lesions recurred and there was no injury to the growth plate or evidence of joint instability. Shortening of $2 \mathrm{~mm}$ to $3 \mathrm{~mm}$ was seen at the distal fibula in comparison with the normal side, but this did not affect the range of movement or the stability of the ankle.
Bone began to regenerate three weeks after resection following the course of the closed periosteal tube (Fig. 2b). Complete healing of the defect occurred at a mean of 4.1 months (3 to 9). In one patient with a closed growth plate bony consolidation was not achieved for nine months but in those with an open growth plate this occurred at a mean of 3.3 months (3 to 4 ).

None of the defects showed evidence of delayed or nonunion. One patient had delayed incorporation of the regenerate at the site of proximal resection after an electric saw had been used to cut the bone ends. No patient had a fracture of the regenerative bone.

At the final follow-up all patients had normal function of the treated limb without discomfort and had resumed their normal activities. Healing was obtained by filling and ossification of the defect.

\section{Discussion}

Aneurysmal bone cysts are benign, non-neoplastic, expansile, osteolytic lesions of multifactorial aetiology, composed of blood-filled spaces separated by connective tissue septa containing fibroblasts, osteoclast-type giant cells and reactive woven bone. ${ }^{12}$ Their precise pathogenesis is unclear, although suggestions include a post-traumatic reaction to reactive vascular malformation or a genetic predisposition. ${ }^{13,14}$

The incidence is 1.4 per 100000 individuals, with $80 \%$ of cases seen before the age of 20 years. The peak incidence is in the second decade of life. ${ }^{15,16}$ They occur more commonly in the metaphysis of long bones, especially the tibia, humerus and femur. ${ }^{6}$

The reported incidence of lesions in the distal fibula varies between $7.1 \%$ and $16.4 \% .^{1,4,17}$ The management of an aneurysmal bone cyst depends on the age of the patient, the location, the size and the aggressiveness of the lesion. ${ }^{9}$ The clinical and pathological behaviour in younger patients does not seem to be more aggressive than that in older children, with no statistically significant differences between the age groups and the rate of recurrence. ${ }^{18,19}$

Since the origin and growth of aneurysmal bone cysts are controversial, a variety of treatments has been described, but a relatively high rate of local recurrence has been recorded depending on the method used. ${ }^{7,9,13,19-22}$ The most common treatments are curettage, resection, intracystic injections and embolisation. ${ }^{13}$ Recurrence is more common in active and aggressive lesions and occurs at a mean of 7.6 months. ${ }^{11,18}$ This has been attributed to incomplete curettage of the primary soft-tissue component because of the reluctance of the surgeon to perform a sufficiently extensive procedure near an open growth plate, especially in the leg. ${ }^{10,21,23} \mathrm{~A}$ mitotic index of seven or more per 50 fields $(\times 750)$ is associated with a higher rate of recurrence than a lower mitotic index. ${ }^{21}$

In aggressive lesions the rate of recurrence using curettage with or without bone grafting varies between $18 \%$ and $59 \% .{ }^{17-19,24}$ Adjunctive therapy such as liquid nitrogen, phenol, the use of polymethylmethacrylate and irradiation 
has been used, but is associated with increased morbidity and no apparent decrease in the rate of local recurrence compared with curettage alone., ${ }^{9,23}$

Marginal en bloc resection may be adopted in an eccentric lesion or in an expandable bone such as the fibula, rib, pubic ramus, metacarpal or metatarsal. This procedure has little morbidity and a minimal risk of recurrence. . $^{3,6,22,25,26}$ Resection of the lesion has the lowest association with recurrence while excision through the margin has consistently decreased the rate of local recurrence. ${ }^{10,27-29}$ Despite the rate of recurrence in various methods of treatments, more aggressive operative intervention does not appear to be indicated and recurrence can be successfully treated by repeated extended curettage and bone graft. ${ }^{19,30}$

It is important to preserve the growth plate, especially in young children, even if the lesions are likely to recur. ${ }^{25}$ When growth of the lower fibular physis is arrested, a valgus deformity of the ankle may develop, the degree of which depends on how many years of potential growth remain. ${ }^{31}$ The risk of secondary valgus deformity is insignificant between ten and 12 years. ${ }^{31}$ If curettage and bone grafting have been performed carefully, the growth plate is unlikely to be affected. ${ }^{2,24}$ In children with an open growth plate, the main problems are at the level of the distal cut with the risk of iatrogenic damage or incomplete resection and the subsequent need for reconstruction. ${ }^{3}$ In younger children, the lesion should be resected at the edge of the growth plate. ${ }^{5}$ All our patients were treated meticulously by resection of the cyst near the edge of the physis without any residual damage.

Recurrence of distal fibular lesions after curettage and bone grafting may occur for which resection is the only successful method of treatment. ${ }^{8}$ An increased morbidity, however, must be considered after such a procedure. ${ }^{9,18}$

The technique used in our study avoided the use of bone graft from the iliac crest, allograft bone or synthetic bone substitutes. Subperiosteal resection has been previously described as a primary method of treatment of lesions of the distal fibula, but there was no regeneration of the bone defect. $^{32}$ Autologous tibial graft has been suggested after total subperiosteal resection of solitary bone cysts of the humerus in order to bridge the defect and to decrease local recurrence. ${ }^{33}$ No previous study has highlighted the effectiveness of the periosteum alone in forming new bone after resection of lesions of the distal fibula. ${ }^{27,32}$ We assume that the inner layer of the periosteum has an osteoblastic capability which allows invasion of the haematoma in the tightly sutured periosteal tube by osteoprogenitor cells. Healing of the remaining defect occurred in a progressive manner which suggests that osteogenesis occurred initially at the margins of the cavity and moved toward its centre over the following weeks. Progressive calcification and ossification followed and the lesion was transformed into a solid bony mass.

The location of the fibular lesion near the ankle may cause instability, recurrence or growth arrest, ${ }^{32}$ but we did not encounter these problems. Although age, the location of the lesion, and its size have been suggested as risk factors for local recurrence in aneurysmal bone cysts, ${ }^{9,22}$ the subperiosteal resection totally removed the pathological lesion. The mean healing time after traditional surgical treatment is 11.6 months (8 to 15 ) whereas after injection with steroids or bone marrow it is 13.9 months (15 to 18$).{ }^{33} \mathrm{In}$ our patients the healing time was much shorter within a mean of 4.12 months (3 to 9).

The main advantage of subperiosteal resection is the complete regeneration of the bone defect and the absence of local recurrence. This can be attributed to the presence of a thick periosteum with substantial regenerative capacity. Preservation of the integrity of the periosteum after subperiosteal resection constitutes a valuable matrix for bone regeneration in children and young adolescents. It plays a major role in the complete filling of the bone defects and avoids the morbidity associated with other techniques.

No benefits in any form have been received or will be received from a commercial party related directly or indirectly to the subject of this article.

\section{References}

1. Capanna R, van Horn JR, Biagini R, et al. Reconstruction after resection of the distal fibula for bone tumor. Acta Orthop Scand 1986;57:290-4.

2. Rizzo M, Dellaero DT, Harrelson JM, Scully SP. Juxtaphyseal aneurysmal bone cysts. Clin Orthop 1993;64:205-12.

3. Rizzo M, Scully SP, Harrelson JM. Ipsilateral fibular slide grafts in the management of distal fibula lesions. Foot Ankle Int 1999;20:135-6.

4. Lampasi M, Magnani M, Donzelli $\mathbf{0}$. Aneurysmal bone cysts of the distal fibula in children: long-term results of curettage and resection in nine patients. J Bone Joint Surg [Br] 2007;89-B:1356-62.

5. Ramirez N, Perez C, Rivera YC. Distal third fibular aneurysmal bone cyst: en bloc resection and proximal third fibular reconstruction. Am J Orthop 2001;30:237-40.

6. Ramirez AR, Stanton RP. Aneurysmal bone cyst in 29 children. J Pediatr Orthop 2002;22:533-9

7. Marcove RC, Sheth DS, Takemoto S, Healey JH. The treatment of aneurysmal bone cyst. Clin Orthop 1995;311:157-63.

8. Lubliner JA, Robbins H, Lewis MM, Present $\mathbf{D}$. Aneurysmal bone cyst of the fibula: en bloc resection with allograft reconstruction. Bull Hosp Jt Dis Orthop Inst 1985:45:80-6.

9. Cottalorda J, Bourelle $\mathbf{S}$. Current treatments of primary aneurysmal bone cysts. $J$ Pediatr Orthop B2006;15:155-67.

10. Campanacci M, Capanna R, Picci P. Unicameral and aneurysmal bone cysts. Clin Orthop 1986;204:25-36.

11. Capanna R, Betteli G, Biaginia R, et al. Aneurysmal bone cysts of long bones. Ital J Orthop Traumatol 1985;11:409-17 (in Italian).

12. Rosenberg AE, Nielsen GP, Fletcher JA. Aneurysmal bone cyst. In: Fletcher CDM, Unni KK, Mertens F, eds. World Health Organization: classification of tumours. pathology and genetics of tumours of soft tissue and bone. Lyon: IARC Press, 2002:338-9.

13. Cottalorda J, Bourelle S. Modern concepts of primary aneurysmal bone cyst. Arch Orthop Trauma Surg 2007;127:105-14.

14. Althof PA, Ohmori K, Zhou M, et al. Cytogenetic and molecular cytogenetic findings in 43 aneurysmal bone cysts: aberrations of 17p mapped to $17 p 13.2$ by fluoresence in situ hybridization. Mod Pathol 2004;17:518-25.

15. Ozaki T, Hillmann A, Linder N. Cementation of primary aneurysmal bone cysts. Clin Orthop 1997;337:240-8.

16. Leithner A, Windhager R, Lang $\mathbf{S}$, et al. Aneurysmal bone cyst: a population based epidemiologic study and literature review. Clin Orthop 1999;363:176-9.

17. Cottalorda J, Kohler R, Sales de Gauzy J, et al. Epidemiology of aneurysmal bone cysts in children: a multicenter study and literature review. J Pediatr Orthop B 2004;13:389-94.

18. Cottalorda J, Kohler R, Chotel F, et al. Recurrence of aneurysmal bone cysts in young children: a multicenter study. J Pediatr Orthop B 2005;14:212-18.

19. Ruiter DJ, Van Rijssel TG, Van Der Velde EA. Aneurysmal bone cysts: a clinicopathological study of 105 cases. Cancer 1977;39:2231-9. 
20. Cole WG. Treatment of aneurysmal bone cysts in childhood. J Pediatr Orthop 1986;6:326-9.

21. Freiberg AA, Loder RT, Heidelberger KP, Hensinger RN. Aneurysmal bone cysts in young children. J Pediatr Orthop 1994;14:86-91.

22. Vergel De Dios AM, Bond JR, Shives TG, et al. Aneurysmal bone cyst: a clinical pathological study of 238 cases. Cancer 1992;69:2921-31.

23. Gibbs CP Jr, Hefele MC, Peabody TD, et al. Aneurysmal bone cyst of the extremities: factors related to local recurrence after curettage with a high-speed burr. J Bone Joint Surg [Am] 1999;81-A:1671-8.

24. Capanna R, Springfield DS, Biagini R, et al. Juxtaepiphyseal aneurysmal bone cysts. Skeletal Radiol 1985;13:21-5.

25. Bollini G, Jouve JL, Cottalorda J, et al. Aneurysmal bone cyst in children: analysis of twenty-seven patients. J Pediatr Orthop B 1998;7:274-85.

26. Biesecker JL, Marcove RC, Huvos AG, Mike V. Aneurysmal bone cysts: a clinicopathologic study of 66 cases. Cancer 1970;26:615-25.
27. Stürz H, Zenker H, Bucki H. Total subperiosteal resection treatment of solitary bone cysts of the humerus. Arch Orthop Trauma Surg 1979;93:231-9.

28. Koskinen EV, Visuri TI, Holmström T, Roukkula MA. Aneurysmal bone cyst: evaluation of resection and curettage in 20 cases. Clin Orthop 1976;118:136-46.

29. Morton KS. Aneurysmal bone cyst: a review of 26 cases. Canadian J Surg 1986;29:110-15

30. Clough JR, Price CHG. Aneurysmal bone cysts: review of twelve cases. J Bone Joint Surg [Br] 1968;50-B:116-27.

31. Wiltse LL. Valgus deformity of the ankle: a sequel to acquired or congenital abnormalities of the fibula. J Bone Joint Surg [Am] 1972;54-A:595-606.

32. Shoji H, Koshino T, Marcove RC. Thompson TC. Subperiosteal resection of the distal portion of the fibula for aneurysmal bone cysts: report of two cases. J Bone Joint Surg [Am] 1970;52-A:1472-6.

33. Baschang A, Von Laer $\mathbf{L}$. Indication and procedure of the operative treatment of benign bone cysts in children and adolescents. Eur J Pediatr Surg 1991;1:207-9. 